\title{
Erratum to: Activities of genes controlling sphingolipid metabolism in human fibroblasts treated with flavonoids
}

\author{
Marta Moskot $^{1}$ - Joanna Jakóbkiewicz-Banecka ${ }^{2}$ • Elwira Smolińska ${ }^{2}$. \\ Bogdan Banecki $^{3}$ - Grzegorz Wegrzyn ${ }^{2}$ - Magdalena Gabig-Cimińska ${ }^{1}$
}

Published online: 22 February 2017

(C) Springer Science+Business Media New York 2017

Erratum to: Metab Brain Dis (2015) 30:1257-1267

DOI 10.1007/s11011-015-9705-x

In the original publication of the article, acknowledgments section was incompletely published. The complete acknowledgments are provided in this erratum.

Acknowledgements

This work was supported by National Science Centre (project grant no. UMO-2011/01/B/NZ1/03686 and N N301 668540). Marta Moskot was funded by National Science Centre project grant no. UMO-2014/12/T/NZ2/00538.

The online version of the original article can be found at http://dx.doi. org/10.1007/s11011-015-9705-x

Magdalena Gabig-Cimińska

m.gabig@biol.ug.edu.pl

1 Laboratory of Molecular Biology (affiliated with the University of Gdańsk), Institute of Biochemistry and Biophysics, Polish Academy of Sciences, Wita Stwosza 59, 80-308 Gdańsk, Poland

2 Department of Molecular Biology, University of Gdańsk, Wita Stwosza 59, 80-308 Gdańsk, Poland

3 Department of Molecular and Cellular Biology, Intercollegiate Faculty of Biotechnology UG-MUG, Kładki 24,

80-822 Gdańsk, Poland 